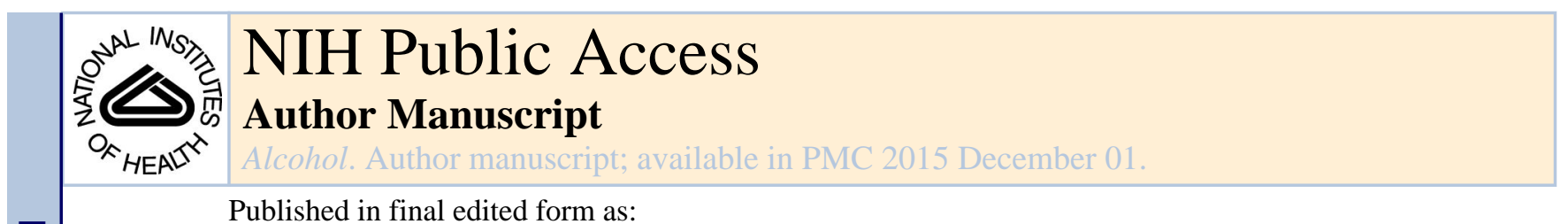

Published in final edited form as:

Alcohol. 2014 December ; 48(8): 765-772. doi:10.1016/j.alcohol.2014.06.009.

\title{
The effect of prior alcohol consumption on the ataxic response to alcohol in high-alcohol preferring mice
}

\author{
Brandon M. Fritz ${ }^{*}$ and Stephen L. Boehm II \\ Indiana Alcohol Research Center, Department of Psychology, Indiana University e Purdue \\ University Indianapolis, Indiana, USA
}

\section{Abstract}

We have previously shown that ethanol-naïve high-alcohol preferring (HAP) mice, genetically predis-posed to consume large quantities of alcohol, exhibited heightened sensitivity and more rapid acute functional tolerance (AFT) to alcohol-induced ataxia compared to low-alcohol preferring mice. The goal of the present study was to evaluate the effect of prior alcohol selfadministration on these responses in HAP mice. Naïve male and female adult HAP mice from the second replicate of selection (HAP2) underwent 18 days of 24-h, 2-bottle choice drinking for 10\% ethanol vs. water, or water only. After 18 days of fluid access, mice were tested for ataxic sensitivity and rapid AFT following a $1.75 \mathrm{~g} / \mathrm{kg}$ injection of ethanol on a static dowel apparatus in Experiment 1. In Experiment 2, a separate group of mice was tested for more protracted AFT development using a dual-injection approach where a second, larger $(2.0 \mathrm{~g} / \mathrm{kg})$ injection of ethanol was given following the initial recovery of performance on the task. HAP2 mice that had prior access to alcohol exhibited a blunted ataxic response to the acute alcohol challenge, but this preexposure did not alter rapid within-session AFT capacity in Experiment 1 or more protracted AFT capacity in Experiment 2. These findings suggest that the typically observed increase in alcohol consumption in these mice may be influenced by ataxic functional tolerance development, but is not mediated by a greater capacity for ethanol exposure to positively influence within-session ataxic tolerance.

\section{Keywords}

Alcohol; Ataxia; Behavior; Ethanol; Genetics; Mouse; Selected lines; Sensitivity; Tolerance; Alcohol consumption

\section{Introduction}

\begin{abstract}
Alcohol use disorders have been demonstrated to have a substantial genetic component (Ducci \& Goldman, 2008; Mayfield, Harris, \& Schuckit, 2008; Schuckit, 2009), with a positive family history having significant predictive value. Recognizing a genetically predisposed state has significant clinical importance; however, the ultimate health concern is
\end{abstract}

(C) 2014 Elsevier Inc. All rights reserved.

* Corresponding author. Department of Psychology, Indiana University - Purdue University Indianapolis, 402 N. Blackford St., LD 301, Indianapolis, IN 46202, USA. Tel.: +1 952994 6683; fax: +1 317274 6756. bmfritz@iupui.edu (B.M. Fritz).. 
whether these individuals, if they choose to consume alcohol, escalate their consumption to dangerous levels (Warner, White, \& Johnson, 2007). Exploring the adaptive processes (i.e., tolerance) that may be positively influenced by alcohol exposure and ultimately, allow these individuals to significantly increase their alcohol consumption over time, will enhance our understanding of how these processes themselves may adapt and drive continued drinking. As it is difficult to account for environmental variables and the subjects' alcohol use history in human studies, animal models offer a powerful approach for addressing this issue.

Numerous lines of rodents have been selectively bred for divergent alcohol intake (i.e., high/ low). Similar to the human literature, studies have shown that these opposite genetic predispositions can associate with highly different responses to alcohol in these lines of rodents (Chester, Lumeng, Li, \& Grahame, 2003; Crabbe, Colville, et al., 2012; Crabbe, Kruse, et al., 2012; Fritz et al., 2014; Fritz, Grahame, \& Boehm, 2013; Grahame, RoddHenricks, Li, \& Lumeng, 2000; Waller, McBride, Lumeng, \& Li, 1983), suggesting that these responses 'genetically correlate' with the alcohol consumption phenotype. In other words, these associations can be indicative of common underlying genes for the response(s) of interest and form of alcohol consumption. One interpretation is that these various responses (ataxia, stimulation, etc.) are components of the complex phenotypes that are high/low alcohol consumption (Crabbe, Phillips, Kosobud, \& Belknap, 1990).

Recently, we have shown that selectively bred high- and low-alcohol preferring mice (HAP and LAP) differ in their sensitivity and capacity to develop acute functional tolerance (AFT) to alcohol's ataxic effects. HAP mice are particularly sensitive to these effects on the ascending limb of the blood ethanol concentration (BEC) curve, as ethanol is being absorbed (Fritz et al., 2013). Furthermore, HAP mice have a significantly greater AFT capacity than do LAP mice. These findings appear to support the alcohol consumption pheno-type of HAP mice because alcohol's intoxicating effects may become salient more rapidly, thereby tightening the temporal relationship between alcohol exposure and its effects. In addition, these mice are able to rapidly ( $<30 \mathrm{~min}$ ) overcome impairment at significantly higher BECs, suggesting greater AFT capacity. The high ethanol intake of HAP mice may reflect, in part, a significant ability to rapidly overcome this alcohol-induced motor impairment, with sustained drinking throughout their active circadian phase in an attempt to achieve an earlier intoxication state that can quickly be masked by AFT.

One question raised by this previous study was whether an alcohol consumption history altered these ataxic responses to ethanol in HAP mice. These lines typically demonstrate an increase in $10 \%$ ethanol intake/preference over days in a continuous-access 2-bottle choice paradigm (Grahame, Li, \& Lumeng, 1999; Matson \& Grahame, 2011; Oberlin, Best, Matson, Henderson, \& Grahame, 2011). Given the already demonstrated impressive rapid AFT capacity in ethanol-naïve HAP mice (Fritz et al., 2013), their capacity to escalate ethanol consumption over days may also be reflective of an enhanced ability for alcohol exposure to positively influence this response. This notion is supported by a previous study demonstrating that the AFT capacity of a mouse line selectively bred to develop a high degree of AFT (HAFT) following acute ethanol administration was augmented by alcohol pre-exposure (Wu, Tabakoff, Szabó, \& Hoffman, 2001). We hypothesized that a history of alcohol consumption would enhance AFT to alcohol-induced ataxia in HAP mice. 


\section{Materials and methods}

\section{Animals}

Naïve adult (postnatal day 60-95) male and female mice from the second replicate of a line selected for high (HAP2) alcohol preference drinking were bred on site at the IUPUI School of Science, Indianapolis, IN (for an in-depth description of the selection process and characterization of these lines, see Oberlin et al., 2011). Three replicates of the HAP/LAP lines now exist and there are extreme differences in alcohol intake and preference with the replicate HAP lines consuming high, intoxicating amounts of alcohol, and replicate LAP lines demonstrating relative avoidance (Oberlin et al., 2011). Only HAP2 mice were used as we previously demonstrated that the genetic differences in ataxic ethanol sensitivity and AFT were present in both replicates 2 and 3 (Fritz et al., 2013). Therefore, testing replicate 3 was deemed unnecessary. Furthermore, the ethanol intake of HAP2 mice is greater and more stable than HAP3 mice (Matson \& Grahame, 2011; Oberlin et al., 2011), perhaps because the selection process is further advanced.

All mice were from generations 43-44 of selection. Animals were singly housed and maintained on a 12-h light/dark cycle with lights on at 2300; testing took place between 2300 and 0500 . Temperature and relative humidity were held constant near $20^{\circ} \mathrm{C}$ and $50 \%$, respectively. Food was available ad libitum and fluids were provided as described below. All experiments were performed under a protocol approved by the IUPUI Institutional Animal Care and Use Committee and were carried out in accordance with the National Institutes of Health Guide for the Care and Use of Laboratory Animals.

\section{Ethanol administration}

One hundred ninety proof ethanol was purchased from Pharmco Inc. (Brookfield, CT) and diluted in sterile $0.9 \%$ physiological saline to a concentration of $15 \% \mathrm{v} / \mathrm{v}$. The solution was freshly mixed each test day. Ethanol was administered via intraperitoneal (i.p.) injection in weight-based doses of $1.75 \mathrm{~g} / \mathrm{kg}$ or $2.0 \mathrm{~g} / \mathrm{kg}$.

\section{Two-bottle choice drinking}

All mice were exposed to the 2-bottle choice drinking procedure, in the same manner in which the lines were selected (Grahame et al., 1999), for 18 days. This duration was chosen because ethanol intake typically plateaus for HAP2 mice by this point (Oberlin et al., 2011). One group of HAP2 mice (E) had access to $10 \%$ (v/v) ethanol and tap water in the home cage via modified $25-\mathrm{mL}$ graduated cylinders, and another group (W) had access to tap water only. Volume readings were taken at 1000, $1 \mathrm{~h}$ before lights-out, every other day, and the designated fluids were replaced. The position of the tubes (left or right side of the cage) was also rotated every other day to avoid the development of a side preference.

\section{Static dowel task}

Details concerning this behavioral assessment have been previously published (Fritz et al., 2013; Grahame et al., 2000). This task was employed to evaluate ethanol-induced ataxia by requiring mice to balance on an elevated wooden dowel horizontally centered in a Plexiglas ${ }^{\circledR}$ box $(32 \times 32 \times 60 \mathrm{~cm} ; 1 \times \mathrm{w} \times$ to prevent falling. Ethanol interferes with the 
ability to perform this task and 'loss of function' (LOF) was declared live by a trained researcher when the majority of the mouse's body was observed to swing below the imaginary horizontal plane that bisects the dowel (Fritz et al., 2013).

\section{Experiment 1: Mellanby (single recovery) approach}

As previously published (Fritz et al., 2013, 2014), this assessment is referred to as the 'Mellanby' approach to AFT quantification as it employs his original conceptualization of the phenomenon, based on observations in canines, by comparing intoxication on the ascending and descending limbs of the time-BEC curve over the course of a single alcohol exposure (Mellanby, 1919). In this manner, AFT can be considered 'within-session tolerance' and is herein referred to as 'M-AFT'. This form of tolerance may be instrumental in the ability of an individual to engage in long, excessive drinking sessions.

Immediately following the fluid reading after the 18th day of 2-bottle choice drinking, mice that had previously had access to ethanol had this tube removed, leaving only water. The other groups had one of their water bottles removed. This was done to ensure that the mice had no ethanol in their systems during the static dowel assessment. At lights-on (2300), mice were moved into the testing room to habituate for $1 \mathrm{~h}$. All animals then received 3 training trials by placing them on the dowel for $1 \mathrm{~min}$ approximately every 5-8 $\mathrm{min}$ to ensure that they could perform the task. Mice were then individually injected with a $1.75 \mathrm{~g} / \mathrm{kg}$ dose of ethanol (i.p.) and immediately placed on the dowel. At the point of LOF, a periorbital blood sample $(25 \mu \mathrm{L})$ was rapidly collected and this value served as the index of sensitivity. Because BEC rises very rapidly following an i.p. injection (on the ascending limb), care was taken to include only samples that were obtained within $8 \mathrm{~s}$ of LOF in the analyses. Mice were then returned to their home cage and retested approximately every 5-8 min until they were able to remain on the dowel for a full minute. Upon recovery (on the descending limb), a second blood sample was collected. Initial sensitivity was quantified as the BEC value at LOF, and AFT was quantified as the difference between BEC at recovery and BEC at LOF (with positive values indicating acute functional tolerance).

\section{Experiment 2: Two-recovery approach}

As M-AFT capacity was evaluated by this task over a relatively short duration (30-60 min post-injection), it was also of interest to evaluate AFT at higher doses of ethanol and over a longer period of time. The 2-recovery procedure of static dowel AFT, originally developed by Erwin and Deitrich (1996), is similar to that of the MAFT approach described above, except no blood sample was taken at LOF and a second $2.0 \mathrm{~g} / \mathrm{kg}$ injection of ethanol was administered following the first recovery. After a 1-h rest period following the second injection, retesting ensued at 5-min intervals until recovery criterion was met again, whereupon another periorbital sinus blood sample was taken. AFT using this 2-recovery approach was calculated by subtracting the BEC value at recovery 1 from the BEC value at recovery 2 .

\section{Blood-ethanol concentration determination}

Blood samples were spun down in a centrifuge and the plasma supernatant was siphoned off and transferred to $0.5-\mathrm{mL}$ micro-centrifuge tubes. Samples were stored at $-80{ }^{\circ} \mathrm{C}$ until 
determination of BEC in mg/dL by an Analox Alcohol Analyzer (Analox Instruments, Lunenburg, MA).

\section{Statistical analysis}

Data were analyzed in the same manner as Fritz et al. (2013) by ANOVAs or repeatedmeasures ANOVAs with all potential factors included. The significance level was set at $p<$ 0.05 and Tukeye-K-ramer or Dunnett's post hoc statistics are reported where relevant. Only the BECs at LOF (Experiment 1) or the BECs at recovery 1 (Experiment 2) were analyzed for group differences. This was done similarly to Fritz et al. (2013) to avoid unnecessary complications brought on by a repeated-measures analysis as the relationship between both BEC values in each experiment is already described by M-AFT or AFT. Statistica 7 software (Statsoft) was used to compute all analyses. Two HAP2 males in the ethanolconsuming group did not demonstrate ethanol preference at any point during 2-bottle choice drinking and were therefore excluded from the analyses. One HAP2 female did not fall from the dowel within 5 min of injection during the ataxia test and was also excluded from those analyses. However, this mouse was included in fluid consumption analyses. Group means split by sex are available in Tables 1 and 2 for Experiment 1 and 2, respectively.

\section{Results}

\section{Experiment 1: Mellanby (single recovery) approach; ethanol consumption}

As is typically found with these lines, female HAP2 mice consumed significantly more ethanol than males over the 18 -day drinking period $(F[1,24]=17.7, p<0.001$; Fig. $1 \mathrm{~A})$. A main effect of day was also detected $\left(F[8192] 1 \frac{1}{4} 2.781, p<0.01\right.$; Fig. 1A), but no day $\times$ sex interaction $(p>0.05)$ was detected. Dunnett's post hoc testing revealed that the E group increased their ethanol intake over days, with consumption on days 3-18 being significantly greater overall than the first reading for days $1-2$ ( $p$ 's < 0.05). In addition, female mice demonstrated significantly greater ethanol preference than males; $F[1,24]=12.849, p<0.01$ (Fig. 1B). A main effect of day $(F[8,192]=8.983, p<0.001)$ also indicated that ethanol preference was significantly greater on days $3-18$ compared to the value from days $1-2$ (Dunnett's $p$ 's < 0.05). Finally, a significant sex $\times$ day interaction $(F[1,24]=8.983, p<0.05)$ also indicated that females demonstrated greater ethanol preference than males on days 7-14 $(p ' s<0.05)$.

\section{Ataxia test}

The mean values for each static dowel task parameter in Experiment 1 are represented for each group/sex combination in Table 1. A main effect of group was found for $\operatorname{LOF}(F[1,50]$ $=18.071, p<0.001 ;$ Fig. $2 \mathrm{~A}$ ) with the ethanol-naïve $\mathrm{W}$ group falling from the dowel significantly sooner after the ethanol injection than the E group. A main effect of sex ( $F$ $[1,50]=6.981, p<0.05)$ was also determined by the observation that female mice fell significantly later than males. A group sex interaction did not reach statistical significance ( $p$ $=0.858)$. The main effect of group for BEC at $\operatorname{LOF}(F[1,50]=8.713, p<0.01 ;$ Fig. $2 \mathrm{~B})$ was in agreement with the behavioral observations at LOF as the same pattern emerged. In other words, an ethanol consumption history in E mice both delayed time to LOF and resulted in higher BECs at this behavioral endpoint. Sex was not a factor in these measures ( $p$ 's >0.05). 
The groups or sexes did not differ in the time taken to recover balance on the dowel ( $p$ 's $>$ 0.05 ; Fig. 2C). Finally, the groups differed in M-AFT ( $F[1,50]=14.346, p<0.001$; Fig. 2D). When examining the M-AFT values, a direct comparison between groups did not seem entirely appropriate as it was unclear whether the E group actually developed M-AFT (value significantly greater than zero). Follow-up t tests comparing each of the group means to zero indicated that the $\mathrm{W}$ group developed significant M-AFT $\left(t_{28}=8.413, p<0.001\right)$, whereas the E group did not $(p>0.05)$. Sex was not a significant factor in analyses of M-AFT (all $p$ 's $>0.05)$.

\section{Experiment 2: Two-recovery approach; ethanol consumption}

Similar to Experiment 1, female E mice consumed significantly more ethanol than males $(F[1,24]=16.407, p<0.001$; Fig. 3A). A main effect of day was also discovered $(F[8,192]$ $=4.204, p<0.001 ;$ Fig. 3A), with Dunnett's post hoc testing indicating that the final ethanol intake measurement for days 17-18 was significantly greater than the initial measurement taken for days 1-2 $(p<0.05)$. Given that the novelty of ethanol access may have influenced consumption measured at the first reading for male mice, with a more stable baseline being represented at the second reading from days 3-4 (Fig. 3A), an overall Dunnett's test for day comparing ethanol intakes to this value instead found significantly greater intake measured on days 11-12 and 15-18 ( $p$ 's $<0.05$ ), collectively suggesting an increase in ethanol intake over the course of the experiment. This supplemental post hoc analysis was justified, considering the absence of a significant $\operatorname{sex} \times$ day interaction $(p=0.285)$. The analysis of ethanol preference also demonstrated significantly greater preference in female mice $(F$ $[1,24]=16.541, p<0.001 ;$ Fig. $3 \mathrm{~B})$. A main effect of day was also detected $(F[8,192]=$ $14.413, p<0.001$; Fig. 3B) with post hoc testing revealing that ethanol preference values calculated from readings on days 9-18 were significantly greater than the preference calculated from values recorded on days $1-2(p$ 's $<0.05)$. The interaction between sex and day was not statistically significant $(p>0.05)$.

\section{Ataxia test}

The mean values for each static dowel task parameter in Experiment 2 are represented for each group/sex combination in Table 2. Following the initial injection of ethanol, the difference between groups in LOF time was replicated $(F[1,44]=9.912, p<0.01$; Fig. 4A), with ethanol-naïve $\mathrm{W}$ mice falling significantly earlier than $\mathrm{E}$ mice. A significant group $\times$ sex interaction $(F[1,44]=5.829, p<0.05)$ suggested that this group difference was largely driven by female mice ( $p<0.05$; Fig. $4 \mathrm{~A}$ and Table 2$)$. A main effect of $\operatorname{sex}(F[1,44]=4.46$, $p<0.05)$ was found for BEC at recovery 1 with females recovering at significantly higher BECs than males (Fig. 4B and Table 2). A group $\times$ sex interaction also approached significance $(F[1,44]=3.929, p=0.054)$, suggesting that a group difference was particularly apparent in female mice. For the repeated-measures analysis of recovery time, main effects of both group $(F[1,44]=26.459, p<0.001$; Fig. $4 \mathrm{C})$ and $\operatorname{sex}(F[1,44]=9.744, p<0.01$; Table 2) were found. Follow-up analyses indicated that both $\mathrm{E}$ and female mice recovered balance on the dowel significantly earlier, overall. A time group interaction was also found $(F[1,44]=10.0, p<0.01)$, indicating that the $\mathrm{E}$ group recovered significantly earlier than the ethanol-naïve $\mathrm{W}$ group following the second injection ( $p<0.05$; Fig. 4C). No factors were significant in the analysis of AFT ( $p$ 's $>0.05$; Fig. 4D). 


\section{Discussion}

With repeated cycles of continuous-access 2-bottle choice drinking, HAP mice typically increase their ethanol intake and preference over days (Grahame et al., 1999; Matson \& Grahame, 2011; Oberlin et al., 2011), and this was generally the case in the current study. Given our previous findings (Fritz et al., 2013), we hypothesized that a prior history of alcohol consumption may enhance the already substantial rapid AFT capacity of HAP2 mice. It was found that an ethanol consumption history produced functional tolerance to ethanol's ataxic effects on the ascending limb of the BEC curve (Fig. 2A and B). However, previous ethanol consumption had no effect on within-session tolerance measures of MAFT or more protracted AFT. Therefore, chronic functional tolerance, but not an increased AFT capacity, may be an important factor in the escalating ethanol consumption of HAP2 mice over the course of continuous-access drinking.

Numerous studies with selectively bred rodent lines have demonstrated that a genetic predisposition for various forms of excessive alcohol consumption is associated with unique responses to alcohol intoxication in naïve animals (Crabbe, Kruse, et al., 2012; Fritz et al., 2013, 2014; Grahame et al., 2000; Waller et al., 1983). Such findings suggest that these baseline responses may contribute to the animals' complex excessive drinking phenotype (Crabbe et al., 1990). Although these baseline responses may be very important for an individual's initial interactions with ethanol, the literature cited above suggests that the various ethanol consumption phenotypes can differentially associate with certain sensitivities and AFT capacities which could potentially be more relevant for a particular consumption phenotype, i.e., greater M-AFT in lines of HAP mice bred for continuous ethanol drinking and blunted sensitivity in HDID-1 mice which preferentially drink ethanol in a binge fashion (Crabbe, Spence, Brown, \& Metten, 2011; Rosenwasser, Fixaris, Crabbe, Brooks, \& Ascheid, 2013). It is therefore important to take the next step to explore how these naïve, baseline responses are affected by a history of ethanol consumption which may drive, in turn, continued or increased ethanol intake over time.

After 2 weeks of free-choice access for $10 \%$ ethanol, P rats demonstrated significant tolerance to ataxia in the 'jump' test (Gatto et al., 1987). The effect of an ethanol consumption history on functional tolerance to ethanol-induced ataxia on a balance beam apparatus has been evaluated in crossed-line high-alcohol preferring mice (cHAP; cross between HAP1 and HAP2 mice). Both 2 and 3 weeks of 2-bottle choice 10\% ethanol consumption were found to produce functional tolerance to balance beam ataxia in cHAP mice induced by a $1.75 \mathrm{~g} / \mathrm{kg}$ injection of ethanol (i.p.), and these observations were not due to metabolic tolerance (Matson, Kasten, Boehm, \& Grahame, 2014). Importantly, these assessments occurred after complete absorption of the ethanol was achieved (on the descending limb). The current study built on the neuro-behavioral phenotype of the HAP family of mice by addressing the effect of an alcohol consumption history on intoxication during the ascending limb, in addition to the descending limb, of the BEC curve, thus providing a clear picture of within-session tolerance or AFT. The results of the current study suggest that 18 days of previous alcohol consumption affected only the ascending limb of this ataxic response in HAP2 mice as the W and E groups did not differ in BEC at recovery 
in Experiment 1 or at either point in Experiment 2 (all of these assessments occurred on the descending limb).

It should be noted that ataxia is a complex construct and the static dowel task only measures one particular facet of motor incoordination. Therefore, a variety of ataxia tests is recommended to achieve a more complete picture of ataxic impairment (Crabbe, Metten, Cameron, \& Wahlsten, 2005). However, our primary goal was to assess how our previous observations in ethanol-naïve HAP mice, using the same static dowel 'Mellanby' and 'TwoRecovery' AFT assessments (Fritz et al., 2013), were influenced by ethanol consumption via the same drinking paradigm employed for selective breeding phenotyping (2-bottle choice for $10 \%$ ethanol or water). In the current study, the observation of blunted sensitivity on the ascending limb is likely indicative of some form of functional tolerance development; however, E mice were not able to develop any greater within-session, AFT to ethanolinduced ataxia than $\mathrm{W}$ mice. Rather, it appears as though an ethanol consumption history negatively influenced M-AFT capacity, likely in large part due to their reduced sensitivity on the ascending limb. Therefore, the development of chronic functional tolerance, rather than AFT capacity, may be a relevant factor in the continued elevation of ethanol intake in HAP2 mice.

Perhaps another interpretation is that ethanol consumption could produce tolerance to AFT mechanisms in HAP2 mice, which is why their established ethanol-naïve M-AFT was no longer apparent in the HAP2E mice. It is not clear how this could potentially factor into the continued, high ethanol intake of these mice. One possibility is that neurobiological alterations induced by this form of ethanol consumption have actually reduced the influence of proteins/systems that regulate ataxic M-AFT, potentially indicating a shift in the neural processes regulating the ataxic response to ethanol. It may therefore be that ataxic withinsession AFT processes are influential in the alcohol drinking of these mice early on, but over time, other responses (perhaps chronic functional tolerance) become more influential.

Our findings may appear somewhat discordant with the previously mentioned study exploring the effect of repeated ethanol exposure on static dowel AFT capacity in HAFT mice (Wu et al., 2001). The researchers evaluated whether 6 days of repeated ethanol injections could alter the AFT capacity of HAFT mice using the 2-recovery approach (Erwin \& Deitrich, 1996). They found that this pre-exposure did not alter the threshold for intoxication (i.e., BEC at LOF), although an increase in AFT capacity and rate was observed. In the present study, we observed the inverse relationship with alcohol preexposure increasing the threshold for intoxication, but reducing M-AFT or having no effect on AFT capacity. Although contrary to what was hypothesized, these findings may not be surprising given that HAFT mice consume relatively little alcohol in a continuous-access paradigm ( 0.5-1.5 g/kg/h) (Erwin, Gehle, \& Deitrich, 2000). Furthermore, the HAP2 mice in the current study voluntarily consumed alcohol (as opposed to experimenter-administered i.p. injections) and also self-administered higher daily doses of alcohol $(\sim 12-22 \mathrm{~g} / \mathrm{kg})$ than the HAFT mice received (3.5-6.0 g/kg), and for a longer period of time (18 days vs. 6 days). Therefore, these qualitatively different alcohol pre-exposures likely differentially influenced ataxic sensitivity and AFT. Because HAP2 mice are specifically bred to voluntarily consume 
high doses of alcohol, our findings may be more directly related to processes governing the escalation of continuous alcohol intake.

Lastly, the observation that the $\mathrm{E}$ group recovered significantly earlier than the $\mathrm{W}$ group after the second injection in Experiment 2, yet displayed equivalent BECs, appears to indicate metabolic tolerance. Four weeks of 2-bottle choice access for $10 \%$ ethanol or water has been previously shown to produce metabolic tolerance in cHAP mice (Matson et al., 2013). HAP2 mice consume significantly less ethanol than cHAP mice in this paradigm (Matson \& Grahame, 2011), and considering that their ethanol consumption duration was only 18 days in the current study, this evidence of metabolic tolerance is impressive and is also the first seen following free-choice drinking in any of the uncrossed HAP lines. Although the E group may have cleared ethanol more quickly than the $\mathrm{W}$ group, this has no bearing on the observed differences in sensitivity or the potential to observe differences in M-AFT or AFT.

In conclusion, a history of ethanol consumption was found to produce functional tolerance to the ataxic response to ethanol on the ascending limb of the BEC curve in HAP2 mice, an animal model of genetic predisposition for excessive, continuous alcohol intake. However, this prior alcohol exposure did not influence within-session AFT development. These findings suggest that the typically observed increase in alcohol consumption in these mice is not mediated by a greater, genetically predisposed capacity for ethanol exposure to positively influence AFT to this form of ataxia, but may instead be influenced by chronic functional tolerance. Future efforts will be aimed at achieving a more complete picture of how voluntary ethanol intake in HAP mice alters ataxia and AFT. In a recent collaboration, our group evaluated ethanol-induced hypnosis on both the ascending and descending limb of the BEC curve in HDID-1 mice using a modified restraint tube apparatus. This approach could further our understanding of the influence of ethanol consumption on adaptive responses to motor impairment.

The current findings highlight the importance of understanding an individual's prior alcohol exposure when assessing ataxic responses. For example, in the human literature, an ataxic measure of alcohol-induced 'body sway' has been used to address the role of genetic predisposition for alcohol abuse (i.e., positive/negative family history for alcohol abuse) on the acute ataxic response to alcohol (Schuckit, 1985). A positive family history of alcohol abuse was found to be associated with reduced ataxic sensitivity, perhaps indicating that these individuals have a blunted response to this form of impairment, which may allow/ encourage them to consume greater quantities of alcohol. This measure has also been used to predict future alcohol abuse likelihood, finding that reduced sensitivity was associated with a greater risk for later abuse (Schuckit, 1994). However, other work has found greater intoxication responses in genetically predisposed individuals (Morzorati, Ramchandani, Flury, Li, \& O'Connor, 2002; Nagoshi \& Wilson, 1987; Newlin \& Thomson, 1990; VogelSprott \& Chipperfield, 1987). Human studies such as these ethically require that individuals have consumed alcohol at some point before testing. In light of the preclinical work discussed above, varying degrees of prior alcohol consumption may influence the nature, or even existence, of these predisposition-associated ataxic responses, perhaps contributing to some degree of study discordance. Studies employing motor responses to acute alcohol as 
predictive tools for alcohol use trajectory, particularly those making comparisons to previously established work, should therefore carefully consider the degree to which subjects have been exposed to alcohol before determining where their findings fit into the literature of alcohol abuse liability biomarkers.

\section{Acknowledgments}

This work was supported by grants AA016789 (SB) and AA07462 (BF) from the NIAAA.

\section{References}

Chester JA, Lumeng L, Li TK, Grahame NJ. High- and low-alcohol-preferring mice show differences in conditioned taste aversion to alcohol. Alcoholism: Clinical and Experimental Research. 2003; 27:12-18.

Crabbe JC, Colville AM, Kruse LC, Cameron AJ, Spence SE, Schlumbohm JP, et al. Ethanol tolerance and withdrawal severity in high drinking in the dark selectively bred mice. Alcoholism: Clinical and Experimental Research. 2012; 36:1152-1161.

Crabbe JC, Kruse LC, Colville AM, Cameron AJ, Spence SE, Schlumbohm JP, et al. Ethanol sensitivity in high drinking in the dark selectively bred mice. Alcoholism: Clinical and Experimental Research. 2012; 36:1162-1170.

Crabbe JC, Metten P, Cameron AJ, Wahlsten D. An analysis of the genetics of alcohol intoxication in inbred mice. Neuroscience \& Biobehavioral Reviews. 2005; 28:785-802. [PubMed: 15642621]

Crabbe JC, Phillips TJ, Kosobud A, Belknap JK. Estimation of genetic correlation: interpretation of experiments using selectively bred and inbred animals. Alcoholism: Clinical and Experimental Research. 1990; 14:141-151.

Crabbe JC, Spence SE, Brown LL, Metten P. Alcohol preference drinking in a mouse line selectively bred for high drinking in the dark. Alcohol. 2011; 45:427-440. [PubMed: 21194877]

Ducci F, Goldman D. Genetic approaches to addiction: genes and alcohol. Addiction. 2008; 103:14141428. [PubMed: 18422824]

Erwin V, Deitrich R. Genetic selection and characterization of mouse lines for acute functional tolerance to ethanol. The Journal of Pharmacology and Experimental Therapeutics. 1996; 279:1310-1317. [PubMed: 8968355]

Erwin VG, Gehle VM, Deitrich RA. Selectively bred lines of mice show response and drug specificity for genetic regulation of acute functional tolerance to ethanol and pentobarbital. The Journal of Pharmacology and Experimental Therapeutics. 2000; 293:188-195. [PubMed: 10734169]

Fritz BM, Cordero KA, Barkley-Levenson AM, Metten P, Crabbe JC, Boehm SL. Genetic relationship between predisposition for binge alcohol consumption and blunted sensitivity to adverse effects of alcohol in mice. Alcoholism: Clinical and Experimental Research. 2014; 38:1284-1292.

Fritz BM, Grahame NJ, Boehm SL 2nd. Selection forhigh alcohol preference drinking in mice results in heightened sensitivity and rapid development of acute functional tolerance to alcohol's ataxic effects. Genes, Brain, and Behavior. 2013; 12:78-86.

Gatto GJ, Murphy JM, Waller MB, McBride WJ, Lumeng L, Li TK. Chronic ethanol tolerance through free-choice drinking in the $\mathrm{P}$ line of alcohol-preferring rats. Pharmacology, Biochemistry, and Behavior. 1987; 28:111-115.

Grahame NJ, Li TK, Lumeng L. Selective breeding for high and low alcohol preference in mice. Behavior Genetics. 1999; 29:47-57. [PubMed: 10371758]

Grahame NJ, Rodd-Henricks K, Li TK, Lumeng L. Ethanol locomotor sensitization, but not tolerance correlates with selection for alcohol preference in high- and low-alcohol preferring mice. Psychopharmacology. 2000; 151:252-260. [PubMed: 10972472]

Matson L, Liangpunsakul S, Crabb D, Buckingham A, Ross RA, Halcomb M, et al. Chronic freechoice drinking in crossed high alcohol preferring mice leads to sustained blood ethanol levels and metabolic tolerance without evidence of liver damage. Alcoholism: Clinical and Experimental Research. 2013; 37:194-201. 
Matson LM, Grahame NJ. Pharmacologically relevant intake during chronic, free-choice drinking rhythms in selectively bred high alcohol-preferring mice. Addiction Biology. 2011; 18:921-929. [PubMed: 22126215]

Matson LM, Kasten CR, Boehm SL 2nd, Grahame NJ. Selectively bred crossed high-alcoholpreferring mice drink to intoxication and develop functional tolerance, but not locomotor sensitization during free-choice ethanol access. Alcoholism: Clinical and Experimental Research. 2014; 38:267-274.

Mayfield RD, Harris RA, Schuckit MA. Genetic factors influencing alcohol dependence. British Journal of Pharmacology. 2008; 154:275-287. [PubMed: 18362899]

Mellanby, E. Alcohol: Its absorption into and disappearance from the blood under different conditions. His Majesty's Stationery Office; London: 1919.

Morzorati SL, Ramchandani VA, Flury L, Li TK, O'Connor S. Self-reported subjective perception of intoxication reflects family history of alcoholism when breath alcohol levels are constant. Alcoholism: Clinical and Experimental Research. 2002; 26:1299-1306.

Nagoshi CT, Wilson JR. Influence of family alcoholism history on alcohol metabolism, sensitivity, and tolerance. Alcoholism: Clinical and Experimental Research. 1987; 11:392-398.

Newlin DB, Thomson JB. Alcohol challenge with sons of alcoholics: a critical review and analysis. Psychological Bulletin. 1990; 108:383-402. [PubMed: 2270234]

Oberlin B, Best C, Matson L, Henderson A, Grahame N. Derivation and characterization of replicate high- and low-alcohol preferring lines of mice and a high-drinking crossed HAP line. Behavior Genetics. 2011; 41:288-302. [PubMed: 20853157]

Rosenwasser AM, Fixaris MC, Crabbe JC, Brooks PC, Ascheid S. Escalation of intake under intermittent ethanol access in diverse mouse geno-types. Addiction Biology. 2013; 18:496-507. [PubMed: 22862671]

Schuckit MA. Ethanol-induced changes in body sway in men at high alcoholism risk. Archives of General Psychiatry. 1985; 42:375-379. [PubMed: 3977555]

Schuckit MA. Low level of response to alcohol as a predictor of future alcoholism. The American Journal of Psychiatry. 1994; 151:184-189. [PubMed: 8296886]

Schuckit MA. An overview of genetic influences in alcoholism. Journal of Substance Abuse Treatment. 2009; 36:S5-S14. [PubMed: 19062348]

Vogel-Sprott M, Chipperfield B. Family history of problem drinking among young male social drinkers: behavioral effects of alcohol. Journal of Studies on Alcohol. 1987; 48:430-436. [PubMed: 3669669]

Waller MB, McBride WJ, Lumeng L, Li TK. Initial sensitivity and acute tolerance to ethanol in the P and NP lines of rats. Pharmacology, Biochemistry, and Behavior. 1983; 19:683-686.

Warner LA, White HR, Johnson V. Alcohol initiation experiences and family history of alcoholism as predictors of problem-drinking trajectories. Journal of Studies on Alcohol and Drugs. 2007; 68:56-65. [PubMed: 17149518]

Wu PH, Tabakoff B, Szabó G, Hoffman PL. Chronic ethanol exposure results in increased acute functional tolerance in selected lines of HAFT and LAFT mice. Psychopharmacology. 2001; 155:405-412. [PubMed: 11441430] 

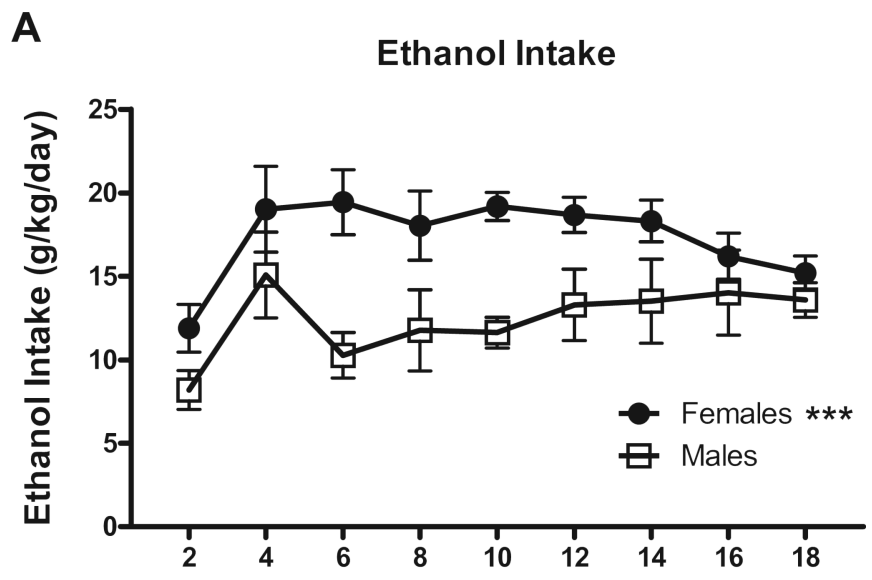

B

Day
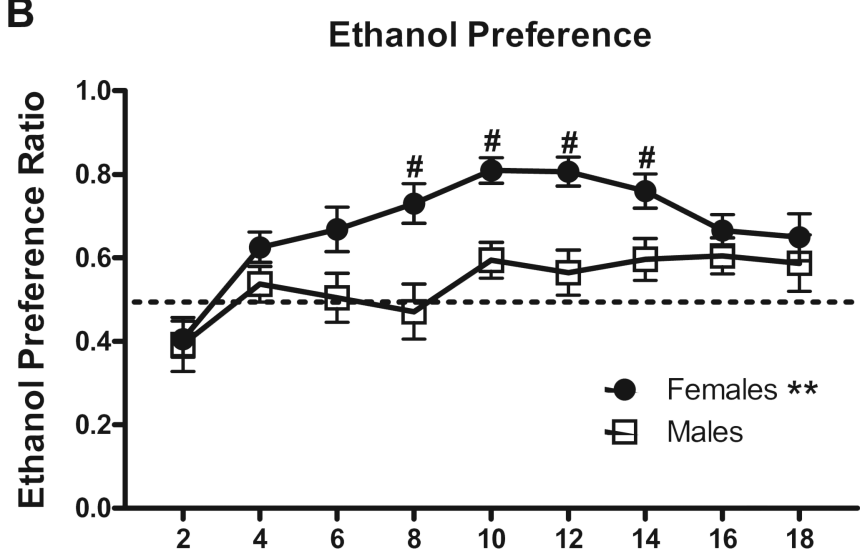

Day

Fig. 1.

Ethanol intake and preference of HAP2 mice in the 2-bottle choice paradigm in which mice had a choice between $10 \%(\mathrm{v} / \mathrm{v})$ ethanol and water in Experiment $1(n=12-14)$. A) Weightbased dose of ethanol consumed, averaged per day. B) Preference index for the volume of $10 \%$ ethanol consumed relative to water. Dashed line indicates an ethanol preference of $50 \%$, with values above this line indicating ethanol preference. $* * p<0.01 ; * * * p 0.001$ for main effect of sex; ${ }^{\#} p<0.05$ vs. males. 


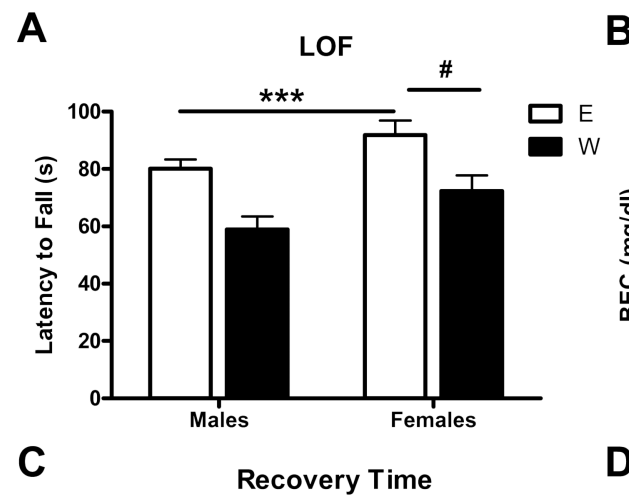

B $\quad$ BEC
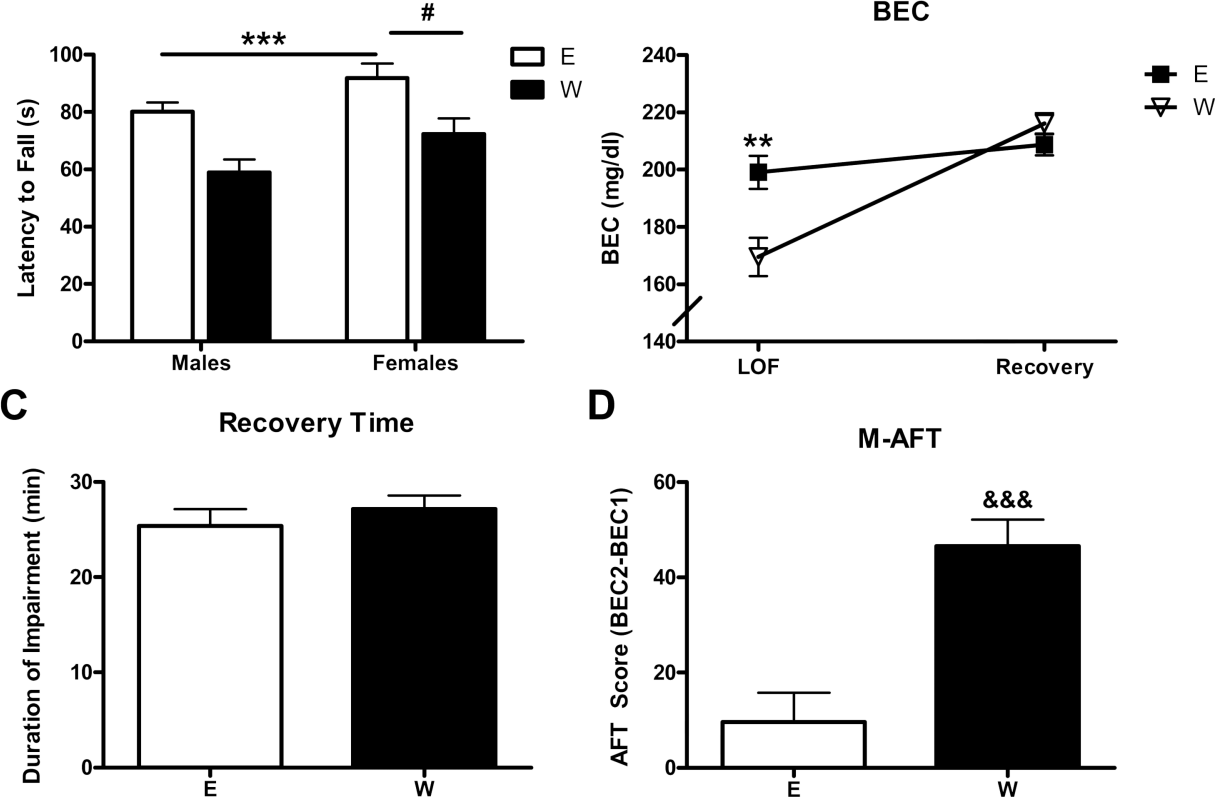

Fig. 2.

Static dowel assessment of sensitivity and M-AFT to ataxia induced by a $1.75 \mathrm{~g} / \mathrm{kg}$ injection of ethanol in Experiment 1 ( $n=12-15$ per group/sex combination). A) Latency to 'loss of function' (LOF) following the ethanol injection. B) BEC values at LOF (intoxication assessment on the ascending limb) and recovery (descending limb). C) Time taken to reach the 1-min recovery criterion following the injection of ethanol. D) M-AFT was calculated as the difference between BEC at recovery and LOF. $* * p<0.01 ; * * * p<0.001 \mathrm{vs}$. W; ${ }^{*} p<$ 0.05 vs. males; $\& \& \& p<0.001$ vs. zero. 
A

Ethanol Intake

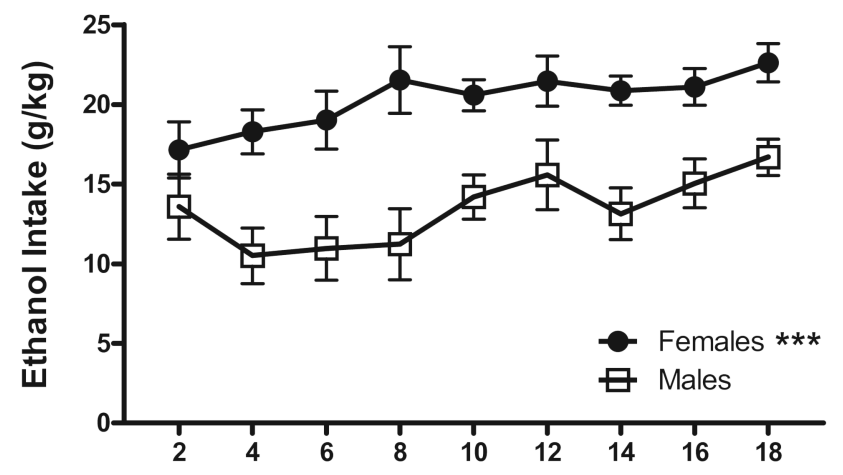

B

Day

B Ethanol Preference

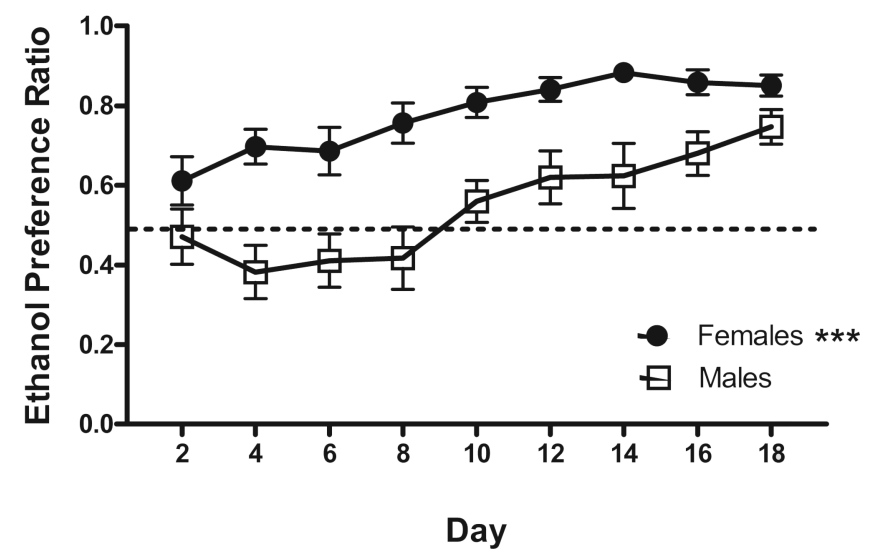

Fig 3.

Ethanol intake and preference in HAP2 mice that had access to both $10 \%$ ethanol and water in Experiment $2(n=13)$. A) Weight-based dose of ethanol consumed, averaged per day. B) Preference index for the volume of $10 \%$ ethanol consumed relative to water. Dashed line indicates an ethanol preference of $50 \%$ with values above this line indicating ethanol preference. ${ }^{* * *} p<0.001$ for main effect of sex. 
A

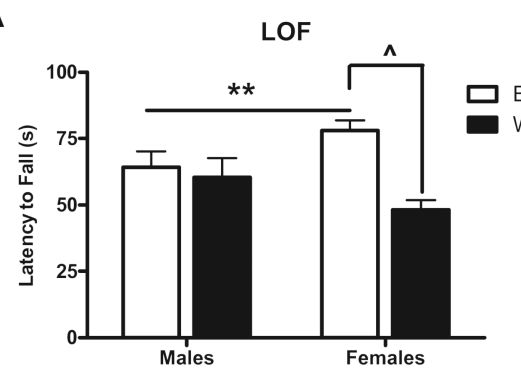

C

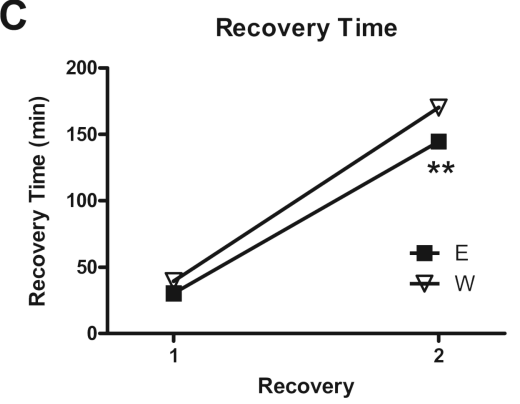

B

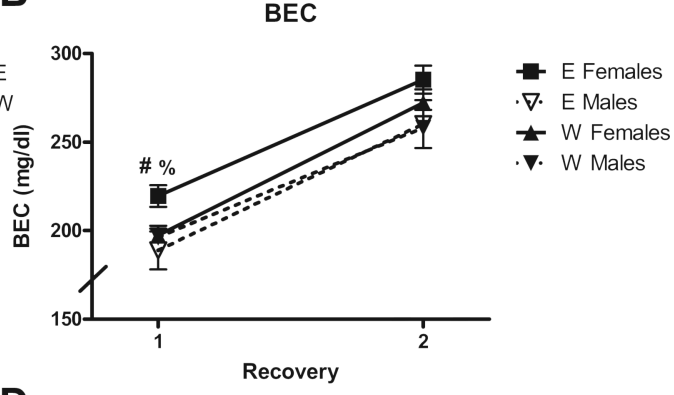

D

AFT

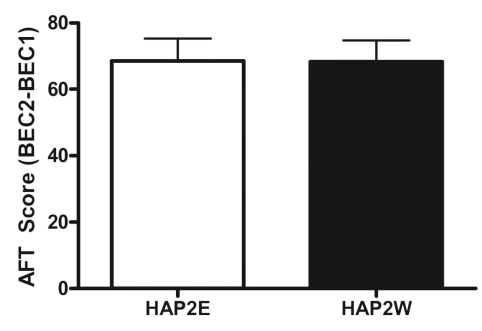

Fig. 4.

Static dowel assessment of AFT to ataxia induced by $1.75 \mathrm{~g} / \mathrm{kg}$ and $2.0 \mathrm{~g} / \mathrm{kg}$ injections of ethanol in Experiment 2 ( $n=11-13$ per group/sex combination). A) Latency to 'loss of function' (LOF) following the ethanol injection. B) BEC values at LOF (intoxication assessment on the ascending limb) and recovery (descending limb). C) Time taken to reach the 1-min recovery criterion following the injection of ethanol. D) AFT was calculated as the difference between BEC at recovery 2 and recovery $1 .{ }^{* *} p<0.001 \mathrm{vs.} \mathrm{W} ;{ }^{\#} p<0.05$ vs. males; ${ }^{\wedge} p<0.05$ for E females vs. $\mathrm{W}$ females; ${ }^{\%} \mathrm{p}=0.053$ for $\mathrm{E}$ females vs. $\mathrm{W}$ females. 


\section{Table 1}

Mean values $( \pm$ SEM) for each measurement using the 'Mellanby' approach in Experiment 1.

\begin{tabular}{llllllll}
\hline Line & Sex & $\boldsymbol{n}$ & LOF $(\mathbf{s e c})$ & Rec. time $(\mathbf{m i n})$ & BEC1 $(\mathbf{m g} / \mathbf{d L})$ & BEC2 $(\mathbf{m g} / \mathbf{d L})$ & M-AFT $(\mathbf{B 2}-\mathbf{B} 1)$ \\
\hline HAP2W & M & 14 & $58.9 \pm 4.5$ & $29.2 \pm 2.2$ & $160.4 \pm 11.0$ & $214.2 \pm 6.0$ & $53.8 \pm 9.1$ \\
& F & 15 & $72.4 \pm 5.4^{\#}$ & $25.3 \pm 1.7$ & $178.1 \pm 7.5$ & $217.9 \pm 4.3$ & $39.9 \pm 6.4$ \\
HAP2E & M & 12 & $80.1 \pm 3.3^{* * *}$ & $25.7 \pm 2.6$ & $189.5 \pm 9.9^{* *}$ & $210.7 \pm 5.9$ & $21.2 \pm 10.4$ \\
& F & 13 & $91.9 \pm 5.1^{\#}$ & $22.5 \pm 1.5$ & $204.4 \pm 8.9$ & $210.6 \pm 4.1$ & $6.2 \pm 9.3$ \\
\hline
\end{tabular}

** $p<0.01$

$* * * x<0.001$ vs. W

${ }_{p}^{\#}<0.05$ vs. males. 


\section{Table 2}

Mean values $( \pm \mathrm{SEM})$ for each measurement using the '2-recovery' approach in Experiment 2.

\begin{tabular}{lllllllll}
\hline Line & Sex & $\boldsymbol{n}$ & LOF $(\mathbf{s e c})$ & Rec. 1 time $(\mathbf{m i n})$ & Rec. 2 time $(\mathbf{m i n})$ & BEC1 (mg/dL) & BEC2 (mg/dL) & AFT (B2-B1) \\
\hline HAP2W & M & 11 & $60.4 \pm 7.3$ & $41.0 \pm 2.0$ & $173.8 \pm 5.6$ & $196.3 \pm 4.9$ & $258.0 \pm 10.2$ & $61.7 \pm 11.5$ \\
& F & 11 & $48.2 \pm 3.7$ & $37.9 \pm 2.3^{\# \#}$ & $166.7 \pm 6.1^{\# \#}$ & $197.3 \pm 5.4^{\#}$ & $272.3 \pm 7.6$ & $75.0 \pm 5.6$ \\
HAP2E & M & 13 & $64.3 \pm 5.9^{* *}$ & $37.1 \pm 3.9^{* *}$ & $153.8 \pm 5.2^{* *}$ & $188.8 \pm 10.7$ & $260.2 \pm 13.5$ & $71.4 \pm 10.0$ \\
& F & 13 & $78.1 \pm 3.8^{\wedge}$ & $23.4 \pm 2.0^{\# \#}$ & $135.4 \pm 4.6^{\# \#}$ & $219.5 \pm 6.1^{\#, \%}$ & $285.3 \pm 7.9$ & $65.7 \pm 9.4$ \\
\hline
\end{tabular}

$* *$

$p<0.001$ vs. W

${ }^{\#}{ }_{p}<0.05$

\#\# $p<0.01$ vs. males

$\wedge$

$p<0.05$ for E females vs. W females

${ }^{\%} p=0.053$ for $\mathrm{E}$ females vs. W females. 